\title{
Analogue Adaptive Filters Using Wave Synthesis Technique
}

\author{
Yan Xie and Bashir M. Al-Hashimi \\ ESD group, School of Electronics and Computer Science, University of Southampton, UK. SO17 1 BJ \\ $\{y x 00 r, b a m h\} @ e c s . s o t o n . a c . u k$
}

\begin{abstract}
This paper describes new analogue adaptive filters based on wave synthesis technique. To facilitate simple adaptation process and stability checks, adaptive series and parallel wave adaptors with only one variable coefficient are developed including a suitable algorithm for updating the coefficients of the filter adaptors. A simulation example of system identification is included confirming the viability of the proposed analogue adaptive filters. An efficient hardware implementation of the analogue adaptive filters is outlined.
\end{abstract}

\section{Introduction}

Analogue adaptive filters have the potential to operate at higher speeds, consume less power and occupy smaller silicon area when compared with adaptive filters implemented using digital techniques [1]. Analogue adaptive filters have been successfully employed in various applications including digital magnetic storage, and it is likely they will continue to play an important role in future digital communication applications. Analogue adaptive filters based on LC ladder prototypes have received particular attention because of their low sensitivity and capability of meeting sharp frequency response specifications [2]. This paper presents a new analogue adaptive filters based on LC ladder prototype. The proposed analogue adaptive filters are derived from the wave synthesis technique developed by Fettweis [3]. The wave synthesis technique was originally developed to design digital filters with low sensitivity. It has been reported that wave filters can also be implemented using analogue technologies, including OTA-C [4], switchedcapacitor (SC) [5] and switched-current (SI) [6-8]. All these reported analogue wave filters have so far been with fixed frequency characteristics. Wave analogue filters with adaptive characteristics have not been addressed in the literature. The aim of this paper is to investigate the explicit design of analogue wave filters for adaptive signal processing.

\section{Analogue wave adaptive filters}

Before we introduce analogue wave adaptive filters, a brief overview of wave theory is given. The wave synthesis technique simulates the behaviour of $L C$ networks by transforming passive inductors or capacitors into one-port elements. Parallel and series wave adaptors are used to connect these one-port elements. An example of a $3^{\text {rd }}$-order low-pass LC ladder prototype is shown in Fig.1(a) with Fig.1(b) showing its equivalent wave filter.

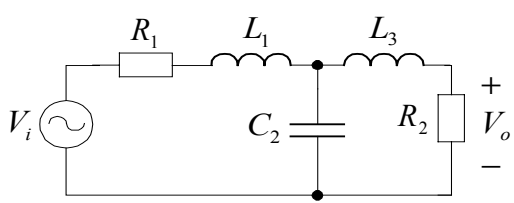

Fig.1(a) $3^{\text {rd }}$-order LC low-pass ladder prototype

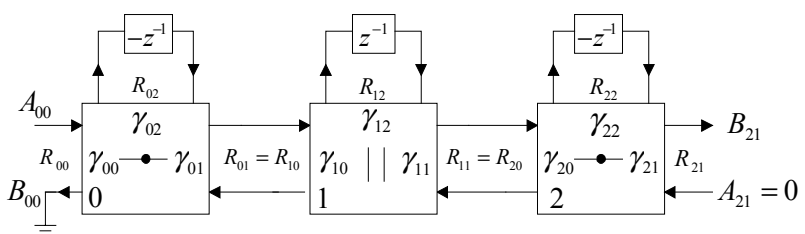

Fig.1(b) its equivalent wave structure

This wave structures consist of three delay cells, two series (numbered 0 and 2) and one parallel (numbered 1) adaptors, where $\gamma_{i j}$ is coefficient of port $j$ in adaptor $i$. The wave adaptor coefficients $\gamma_{i j}$ are calculated using the following expressions, which must be satisfied for each 3 port adaptor:

$$
\sum_{j=0}^{2} \gamma_{i j}=2, \quad \text { where } i=0,1,2
$$

3-port series adaptors: $\quad \frac{\gamma_{i 0}}{R_{i 0}}=\frac{\gamma_{i 1}}{R_{i 1}}=\frac{\gamma_{i 2}}{R_{i 2}}$

3-port parallel adaptors: $\frac{\gamma_{i 0}}{G_{i 0}}=\frac{\gamma_{i 1}}{G_{i 1}}=\frac{\gamma_{i 2}}{G_{i 2}}$

where $R_{i j}$ and $G_{i j}$ are the port resistance and conductance, respectively, of port $j$. They are calculated from the LC prototype: $R_{00}=R_{1}=1, \quad R_{02}=2 L_{1} / T$, $R_{12}=T / 2 C_{2}, R_{22}=2 L_{3} / T, R_{21}=R_{2}=1$ ( $T$ is sampling period). The other port resistances are decided by their corresponding wave adaptor coefficients $\gamma_{i j}$, which are often chosen to optimise the circuit of analogue wave filter [8]. The frequency response of wave filters is dependent on the values of the wave adaptor coefficients $\gamma_{i j}$. Therefore, wave filters can be made adaptive by updating each set of wave adaptor coefficients $\left(\gamma_{i j}, i=0,1,2\right)$ toward their required values. However, when wave adaptor coefficients, for example $\gamma_{00}$ and $\gamma_{01}$ in wave adaptor0, are adapted, the port resistance $R_{01}$ will change as well according to Eq.(3).

$$
\frac{\gamma_{00}}{R_{00}}=\frac{\gamma_{01}}{R_{01}}, \text { where } R_{00}=1
$$

Since port10 and port01 are the same port (Fig.1), this means port resistance $R_{10}$ should be the same as $R_{01}$. 
Consequently, $R_{10}$ will change with $R_{01}$ which will result in the variation of wave adaptor 1 coefficients according to Eq.(4).

$$
\frac{\gamma_{10}}{G_{10}}=\frac{\gamma_{11}}{G_{11}}, \text { where } G_{10} \text { is inverse of } R_{10}
$$

So if we adapt the coefficients of wave adaptor0, it will affect the values of the coefficients in wave adaptorl. This conflicts the design of wave adaptive filters, whose wave adaptor coefficients are required to be varied independently of each other towards their required values. Therefore, in order to achieve wave adaptive filters, it is necessary to fix the value of interface port resistance $\left(R_{01}\right.$ and $R_{11}$ in this example) during the adaptation process. From Eq.(3) and Eq.(4), it can be achieved by keeping the corresponding coefficients in each wave adaptor at the fixed ratio during the adaptation process. For example, in order to fix the value of port resistance $R_{01}=m$, $(m=1,2,3 \ldots)$ in wave adaptor0, the wave adaptor coefficients $\gamma_{01}$ should be kept as $m \cdot \gamma_{00}$ during the wave adaptor0's adaptation process. To achieve the above adaptation process, new series and parallel wave adaptors are developed. Setting $m=1$ (this will simplify the adaptive series and parallel wave adaptors) and substituting $R_{01}=R_{11}=m$ into Eq.(2), the following relationship is obtained:

$$
\gamma_{i 0}=\gamma_{i 1}, i=0,1,2
$$

And the input-output relationship of 3-port series and parallel wave adaptor are:

Series adaptor:

$$
\left[\begin{array}{c}
B_{i 2} \\
B_{i 1} \\
B_{i 0}
\end{array}\right]=\left[\begin{array}{ccc}
2 \gamma_{i 0}-1 & 2 \gamma_{i 0}-2 & 2 \gamma_{i 0}-2 \\
-\gamma_{i 0} & 1-\gamma_{i 0} & -\gamma_{i 0} \\
-\gamma_{i 0} & -\gamma_{i 0} & 1-\gamma_{i 0}
\end{array}\right] \cdot\left[\begin{array}{c}
A_{i 2} \\
A_{i 1} \\
A_{i 0}
\end{array}\right]
$$

Parallel adaptor:

$$
\left[\begin{array}{c}
B_{i 2} \\
B_{i 1} \\
B_{i 0}
\end{array}\right]=\left[\begin{array}{ccc}
1-2 \gamma_{i 0} & \gamma_{i 0} & \gamma_{i 0} \\
2-2 \gamma_{i 0} & \gamma_{i 0}-1 & \gamma_{i 0} \\
2-2 \gamma_{i 0} & \gamma_{i 0} & \gamma_{i 0}-1
\end{array}\right] \cdot\left[\begin{array}{c}
A_{i 2} \\
A_{i 1} \\
A_{i 0}
\end{array}\right]
$$

According to the above relationships, signal flow graphs (SFG) have been derived for the series and parallel adaptive adaptors, as shown in Fig.2.

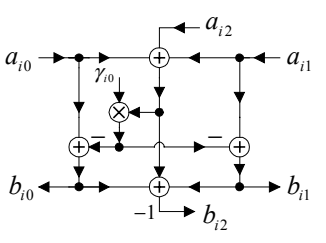

(a)

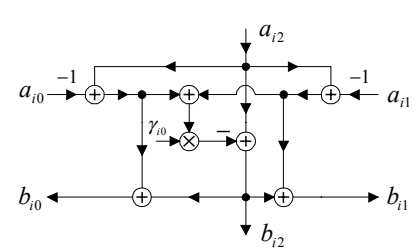

(b)
Fig.2 adaptive series and parallel wave adaptors

Compared with the analogue wave filter with fixed character [8], analogue wave adaptive filters, which based on these adaptors, can keep the interface port resistance fixed during adaptation process and update each coefficient independently toward its required value. In addition, Fig. 2 shows each wave adaptor requires only one coefficient to be varied for the adaptive operation, for example, $\gamma_{00}$ for wave adaptor0.

From the definition of wave adaptor coefficients, Eq.(2), each set of wave adaptor coefficients corresponds to the passive component (for example, the coefficients, $\gamma_{0 j}, j=0,1,2$, in wave adaptor0 corresponds to $L_{1}$, Fig.1.). Therefore, updating the adaptor coefficients during adaptation process, the wave adaptive filter behaves like an adaptive LC filter. Furthermore, due to this correspondence, the wave structure has simple stability checks. The stability of the wave structure can be easily guaranteed by ensuring the values of passive components $L$ and $C$ not to be negative. From Eq.(1) and (2), it can be shown that the wave adaptive filter structure is stable, provided the wave adaptor coefficients satisfy the following conditions:

$$
0<\gamma_{i 0}<1, i=0,1,2
$$

\section{Adaptive algorithm}

To implement analogue wave adaptive filters, an adaptive algorithm is required to update the filter's adaptor coefficients. The performance criteria used for adaptive filter is the mean-squared error $(M S E)$ :

$$
\varepsilon(p)=E\left[(d-y)^{2}\right]=E\left[e^{2}\right]
$$

where $p$ is adaptive parameter, $d$ is desired output, $y$ is filter output and $E[]$ denotes expectation.

The general iterative update rule is [9]:

$$
p(k+1)=p(k)-\mu \cdot \nabla_{p(k)} \varepsilon(p(k))
$$

where $\mu$ is constant parameter determining the rate of adaptation, $p(k)$ is the value of adaptive parameter at $k t h$ iteration and $\nabla_{p(k)} \varepsilon(p(k))$ is gradient estimation. The well-known LMS algorithm takes instantaneous squared error, $e^{2}(k)$, as its expected value, whose resulting gradient estimation is:

$$
\nabla_{p(k)} \varepsilon(p(k))=\nabla_{p(k)}\left(e^{2}(k)\right)=-2 e(k) \nabla_{p(k)} y(k)
$$

For adaptive state-space filter and the adaptive direct-form IIR filter, the gradient signal $\nabla_{p(k)} y(k)$ is obtained through the sensitivity formulas [2,9]. In wave adaptive filter, the input-output transfer function is expressed in terms of wave adaptor coefficients [8], and it is usually in a complicated form. So its gradient estimate functions will be more complicated and difficult to implement. Therefore, an alternative approach should be used to estimate the gradient signal in wave adaptive filter. Recently, a new algorithm for filter adaptation, called the dithered linear search (DLS) technique, has been introduced [10]. The application of DLS algorithm to the wave adaptive filter is considered now. The term dither here refers to the action of perturbing the wave adaptor coefficients $\gamma_{i 0}$ by intentionally injecting small random (or pseudorandom) signal with zero mean, $\delta_{i}$. The wave gradient signal is given by measuring the change of squared-error function with respect to the dithered signal. The binary dither signal $\delta_{i}$ with small amplitude $\Delta$ and 
zero mean is expressed as:

$$
\delta_{i}(k)= \pm \Delta, E\left[\delta_{i}(k)\right]=0
$$

The estimation equation for gradient signal is derived as follows:

$$
\begin{aligned}
& \frac{\partial \varepsilon}{\partial \gamma_{i 0}}=\frac{\varepsilon\left(\left[\gamma_{00}, \gamma_{10} \cdots \gamma_{i 0}+\Delta \cdots\right]^{T}\right)-\varepsilon\left(\left[\gamma_{00}, \gamma_{10} \cdots \gamma_{i 0}-\Delta \cdots\right]^{T}\right)}{2 \Delta} \\
& =\frac{1}{\Delta^{2}} \cdot E\left[\delta_{i}(k) \cdot e^{2}(k)\right] \approx \frac{1}{\sigma^{2}} \cdot \delta_{i}(k) \cdot e^{2}(k)
\end{aligned}
$$

where $\sigma^{2}$ is the variation of the dither signal

From Eq.(8), the update operation for the wave adaptor coefficient is given as follows:

$$
\gamma_{i 0}(k+1)=\gamma_{i 0}(k)-\frac{\mu}{\sigma^{2}} \cdot \delta_{i}(k) \cdot e^{2}(k)
$$

A block diagram is shown in Fig.3 for the adaptation operation of each series or parallel wave adaptor coefficient.

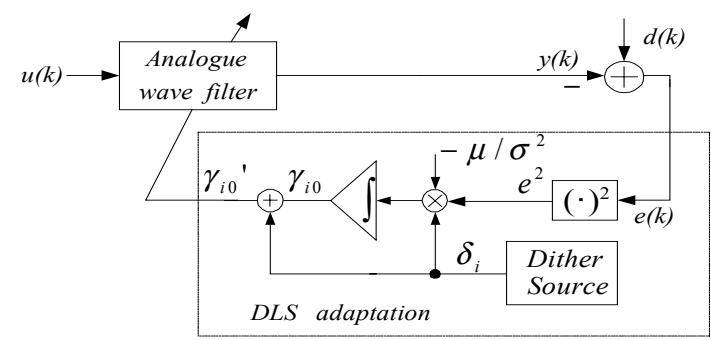

Fig.3 adaptation process using DLS algorithm

\section{Simulation and application}

In this section, the theoretical analysis of the wave adaptive analogue filters is validated using Matlab and is used in a system identification application. In order to model the adaptation process in Matlab, it is necessary to model the adaptive filter and DLS algorithm. The wave adaptive filter consists of adaptive series and parallel adaptors and delay cells. The model of the wave adaptive structure is derived by describing the wave adaptors using Eq.(5). The DLS update process, shown in Fig.3, is modelled and simulated by describing Eq.(12). A dither signal producer was implemented by a random binary function with zero mean and variance of $\sigma^{2}$ and the constraints of Eq.(6) are employed to ensure stability. Replication of the DLS block diagram is necessary for each coefficient. One common application of adaptive analogue filters is system identification. Fig.4 shows the block diagram of system identification model. As an example of all-pole filters, $3^{\text {rd }}$-order lowpass Chebyshev wave filter with $0.177 \mathrm{~dB}$ ripple, and 0.2 normalized cutoff frequency has been chosen as reference filter. The normalized value of passive components in the prototype circuit, Fig.1(a), are calculated as: $L_{1}=L_{3}=0.8183 \mathrm{H}$, $C_{2}=0.7943 \mathrm{~F}$. Using the wave synthesis technique [3], the coefficients of the reference wave filter are: $\gamma_{00}=\gamma_{01}=0.55, \quad \gamma_{02}=0.9, \quad \gamma_{10}=\gamma_{11}=0.5573$, $\gamma_{12}=0.8854, \gamma_{20}=\gamma_{21}=0.55, \gamma_{22}=0.9$

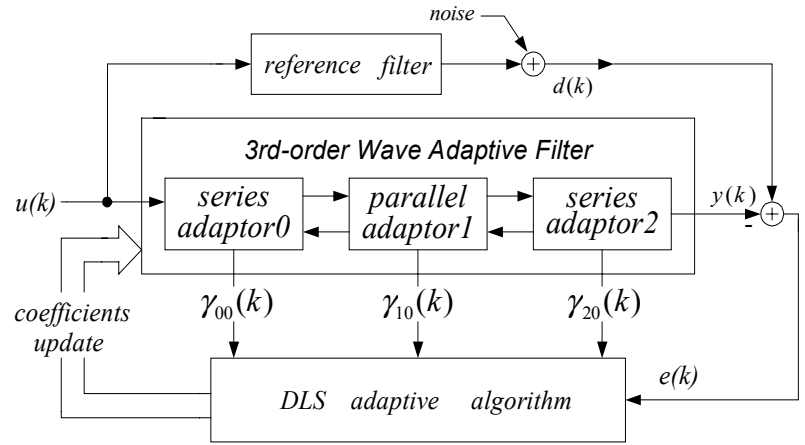

Fig.4 block diagram of system identification

It is assumed that the wave adaptive filter is also based on a $3^{\text {rd }}$-order wave structure. According to adaptive theory [9], both the wave adaptive filter and the system to be identified are fed with an input white signal of variance 5. White noise of variance 0.1 is added to the output of the reference filter. The DLS algorithm converge factor $\mu$ used in this example is $4 \times 10^{-4}$. For each data sample $k$, $1<k<2000$, a dither signal with variance of $4 \times 10^{-3}$ is inserted into the system, Fig.3. Mean-squared error (MSE) is used as the performance criteria for the DLS adaptation.

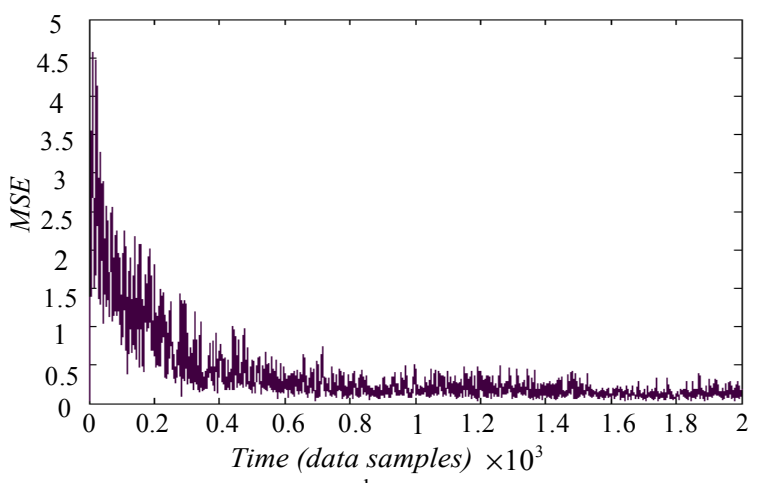

Fig.5 learning curves of $3^{\text {rd }}$-order wave adaptive filter

Results from an ensemble of 40 simulation runs are plotted in Fig.5. The simulated learning curve the MSE converges to its minimum value, which demonstrates the validity of the proposed structure. The steady values of wave adaptor coefficients, after 2000 samples, are:

$\begin{array}{ll}\gamma_{00}(2000)=\gamma_{01}(2000)=0.5325, & \gamma_{02}(2000)=0.935, \\ \gamma_{10}(2000)=\gamma_{11}(2000)=0.5637, & \gamma_{12}(2000)=0.8726, \\ \gamma_{20}(2000)=\gamma_{21}(2000)=0.5341, & \gamma_{22}(2000)=0.9318\end{array}$ These coefficients compare well with those of the reference filter (within 3\% difference with reference value), indicating that the wave adaptive filter is operating as expected. The frequency response of the adaptive and reference filter are given in Fig.6 showing after the adaptation process, the wave adaptive filter exhibits similar performance to the ideal reference filter, indicating successful system identification. 


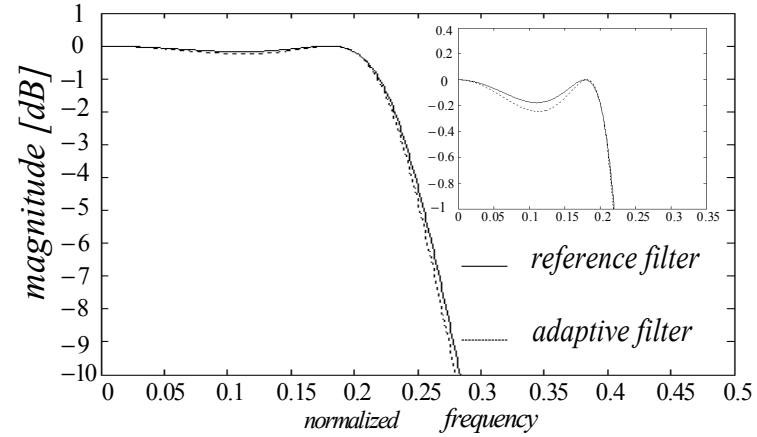

Fig.6 frequency response of reference and wave adaptive filter

\section{Circuit implementation}

Wave adaptive filters have two parts: the analogue wave filter and the DLS adaptation part, Fig.3. Different analogue technologies, such as switched-capacitor (SC) and switched-current (SI), can be used to implement the part of analogue wave filter circuit. SI technology is more suitable for implementing the part of analogue wave filter because the values of wave adaptor coefficients depend only on the aspect ratio of the current mirror transistors in SI technology [8]. Previous research has shown that nonideal characteristics, i.e. dc-offset, is introduced into the adaptive filter by using analogue implementation of adaptation part [1]. It will lead to deterioration in the performance of analogue adaptive filters. Recently there is indication that the digital implementation of gradient algorithm is more robust in the presence of dc-offset $[1,10]$. Therefore, the hardware-efficient method for digitally adapting analogue SI wave filter structure is considered here, Fig.7.

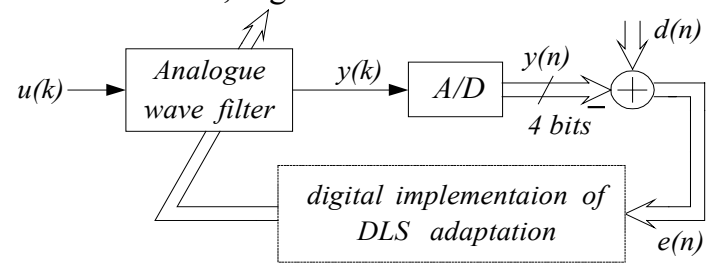

Fig.7 analogue wave adaptive filter

An $\mathrm{A} / \mathrm{D}$ converter is used to converter discrete-time output signal $y(k)$ to digital signal $y(n)$. The 4 bits digital signal is employed for parallel digital signal processing. The DLS algorithm is performed through digital implementation, which is free of non-ideal characteristics of analogue circuit.

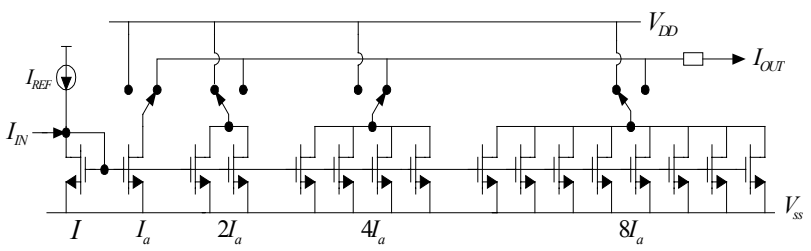

Fig.8 current mirror with adaptable output $\left(I=16 I_{a}\right)$

The analogue wave filter with variable frequency response can be realized through the implementation of series and parallel adaptor with adaptable wave adaptor coefficient $\gamma_{i 0}$, Fig.2. The value of wave adaptor coefficient depends on the aspect ratio of the current mirror transistors. The variation of coefficient values in series or parallel adaptors can be achieved with an array of unit-size transistors through a set of digitally controlled MOS switches, Fig.8.

\section{Conclusion}

This paper has demonstrated the feasibility of using the wave synthesis technique to design analogue adaptive filters. This has been achieved by developing adaptive series and parallel adaptors with only one coefficient per adaptor that needs to be updated. This has the potential to meet two desirable features in adaptive signal processing: simple adaptation process and easy stability checks. A simulation of system identification has been included, validating the theoretical analysis of the proposed analogue adaptive filters. Furthermore, an outline of a hardware implementation of the filters has been presented.

Acknowledgements: The authors wish to thank EPSRC for funding this work, under grant number GR/N31900.

\section{Reference:}

[1] A. Carusone and D.A. Johns, 'Analogue adaptive filters: past and present', IEE Proceedings of Circuits, Devices\&Systems, Vol.147, No.1, pp. 82-90, Feb. 2000.

[2] K.A. Kozma, D.A. Johns, and A.S. Sedra, 'Automatic tuning of continuous-time integrated filters using an adaptive filter technique', IEEE Trans. On Circuits and Systems, vol. 38, No.11, pp. 337-343, Nov. 1991

[3] A. Fettweis, 'Wave digital filter: theory and practice', Proc. IEEE, 74, (2), pp. 270-327, 1986

[4] J. Tingleff and C. Toumazou, 'Integrated current mode wave active filters based on lossy integrators', IEEE Trans. On Circuits and Systems, vol. 42, No.5, pp. 237243, May, 1995

[5] U.Kleine, 'Design of wave-SC filters using building blocks', Int. Journal Circuit Theory and Appl., vol. CT12, pp. 69-87, 1984.

[6] A. Rueda, A.Yufera, and J.L Huertas, 'Wave analogue filters using switched current techniques', Electronics Letters, vol.27, no.16, pp. 1482-1483, 1991.

[7] J.D, Lancaster, B.M. Al-Hashimi, and M. Moniri, 'Efficient SI wave elliptic filters based on direct and inverse Bruton transformations', IEE Proceedings of Circuits, Devices\&Systems, Vol.146, No.5, pp. 235-241, Oct. 1999.

[8] C. Toumazou, J.B., Hughes, and N.C. Battersby, "Switched currents: an analogue technique for digital technology", Peter Peragrinus Ltd, 1993.

[9] B. Widrow and S. D. Stearns, 'Adaptive signal processing', Prentice Hall 1985

[10] A. Carusone 'Digital algorithms for analog adaptive filters', Ph.D dissertation, University of Toronto, Canada, 2002. 